\title{
An empirical assessment of different kernel functions on the performance of support vector machines
}

\author{
Isaac Kofi Nti, Owusu Nyarko-Boateng, Felix Adebayo Adekoya, Benjamin Asubam Weyori \\ Department of Computer Science and Informatics, University of Energy and Natural Resources, Sunyani, Ghana
}

\begin{tabular}{l} 
Article Info \\
\hline Article history: \\
Received Apr 16, 2021 \\
Revised Sep 11, 2021 \\
Accepted Oct 22, 2021 \\
\hline
\end{tabular}

\section{Keywords:}

Gaussian radial basis function

Kernel function

Machine learning

Support vector machine

\begin{abstract}
Artificial intelligence (AI) and machine learning (ML) have influenced every part of our day-to-day activities in this era of technological advancement, making a living more comfortable on the earth. Among the several AI and ML algorithms, the support vector machine (SVM) has become one of the most generally used algorithms for data mining, prediction and other (AI and ML) activities in several domains. The SVM's performance is significantly centred on the kernel function (KF); nonetheless, there is no universal accepted ground for selecting an optimal KF for a specific domain. In this paper, we investigate empirically different KFs on the SVM performance in various fields. We illustrated the performance of the SVM based on different KF through extensive experimental results. Our empirical results show that no single KF is always suitable for achieving high accuracy and generalisation in all domains. However, the gaussian radial basis function (RBF) kernel is often the default choice. Also, if the KF parameters of the RBF and exponential RBF are optimised, they outperform the linear and sigmoid KF based SVM method in terms of accuracy. Besides, the linear KF is more suitable for the linearly separable dataset.
\end{abstract}

This is an open access article under the CC BY-SA license.

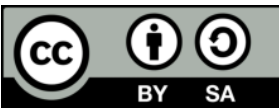

\section{Corresponding Author:}

Isaac Kofi Nti

Department of Computer Science and Informatics

University of Energy and Natural Resources

Sunyani, Ghana

Email: isaac.nti@uenr.edu.gh

\section{INTRODUCTION}

In the $21^{\text {st }}$ century, artificial intelligence (AI) and its sub-disciplines such as machine learning (ML), data mining, deep learning and expert systems have experienced a rebirth following parallel developments in computer power, theoretical understanding and vast amounts of data. These techniques have taken centre stage in the technology industry, assisting in solving several thought-provoking computing, software engineering, and operations research issues [1], [2]. They have impacted demand forecasting, financial analysis, supply chain planning, computer vision, big data analytics, customer engagement, business domain knowledge, education and many more domains. Several ML algorithms like decision trees (DTs), neural networks $(\mathrm{NN})$, K-nearest neighbour $(\mathrm{KNN})$, naïve base $(\mathrm{NB})$, random forest $(\mathrm{RF})$ and support vector machine (SVM) have achieved real-world application success [3]. Among these algorithms, the SVM has been widely used recently. To mention a few, finance [4], [5], engineering [6], healthcare [7]-[9].

The SVM is one of the most robust supervised ML algorithms based on statistical learning theory (STL) developed by Vapnik [10]. The SVM employs the risk minimisation theory to establish the best separation hyperplane in multi-dimensional space to classify a bipartite outcome [11]. Initially, the SVM was designed for binary classification [12]; however, of late, the SVM is applicable for both classification and 
regression ML tasks. The performance of the SVM has been compared with other ML algorithms, such as Bayesian logistic regression, and decision tree [13], [14], random forest [15], [16], neural network [17], [18] and k-nearest neighbours [19], [20]. Notwithstanding variations in the experimental outcomes, the SVM is equated to more traditional models in many of these studies. In contrast, other studies [13], [14], [16], [19] reported that the SVM grippingly outperformed several conventional models.

When training an SVM model, there is a need to choose a KF and its associated parameters, and this is one of the biggest challenges to users of the SVM [5], [11], [21]. The KF permits the SVM (a linear machine) to transform the feature space and act as a non-linear model. The KF parameters regulate the shape of the separating margin used to classify a set of features. An accurate selection of these parameters can significantly improve the prediction accuracy of the SVM [4], [22]. However, the availability of numerous KFs makes it challenging to select the appropriate one for a specific domain. Unfortunately, several researchers (especially beginners) adopt default SVM without worrying about the parameters it uses (e.g., KF).

Nonetheless, selecting all these parameters is necessary before using the SVM in a specific task since they are task-dependent. Besides, there is no universal accepted technique for choosing the appropriate KF and its parameters in a particular domain to attain high generalisation [12]. Additionally, the optimal regularisation parameter (C) is pivotal to obtain accurate outcomes [4], [17]. Hence, the current study presents a comprehensive comparative analysis of several KF on SVM performance for heart disease detection, exchange rate, and weather prediction. Furthermore, we aim to make the KF generalised for a specific domain.

Based on the above discussions, this study seeks to answer the question; which KF and its parameters are suitable for achieving higher generalisation of the SVM under a given dataset? We hypothesised that no single KF and its associated parameters are suitable for all domain applications. The rest of this paper is organised as shown in. First, section 2 presents the basic overview of the SVM, the various types of KF and the study framework. Then, we present the empirical results and discussions in section 3, followed by the study conclusions and future works in section 4.

\section{RESEARCH METHOD}

\subsection{Machine learning}

ML is a subfield of AI centred on creating computer algorithms capable of analysing and learning data with intrinsic patterns and improving their accuracy rate with time without independently. According to [23], ML can be defined graphically, as shown in Figure 1. Given some class of tasks $(T)$, to a computer program, the program is said to learn from experience $(E)$ and performance measure $(P)$, if its performance at $(T)$, as measured by $\mathrm{P}$, improves with $(E)$. Typically, ML is divided into four areas [24], i.e., 1) supervised learning (SL), 2) unsupervised learning (UL), 3) evolutionary learning (EL) and 4) reinforcement learning (RL). Among these four techniques, studies [3], [25], [26] shows that SL is the most commonly used by researchers and professionals due to the already labelled dataset's availability and SL lesser computational time than UL, RL and EL.

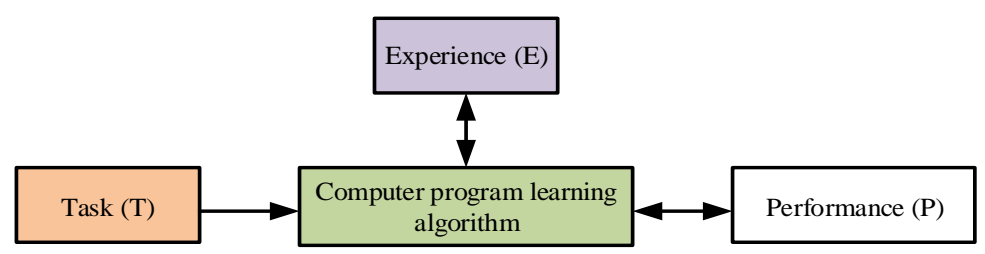

Figure 1. ML definition

Several SL algorithms exist, e.g., KNN, linear regression, RF, logistical regression, gradient boosted trees (GBT), NN, SVM, DT and NB. However, since the current study centres on the SVM, we briefly present its overview in the subsequent section.

\subsection{Support vector machine}

The SVM algorithm was developed at AT\&T Bell laboratories [10] to accurately classify binary dependent features using a unique hyperplane $(\mathrm{H})$. The SVM maps input features $(v)$ to a higher dimensional space where the best separating hyperplane is created. The hyperplane serves as a borderline sandwiched between two classes, created by maximising the margin between support vectors of both classes [19]. Typically, the hyperplane differs in value for different feature dimensions, e.g., for 1-dimension, $\mathrm{H}$ would be a point; for 2-dimension, $\mathrm{H}$ would be a straight-line. Whiles for dimensions greater than two, $H$ would be a plane. Figure 2 shows a simple SVM [27]. The H with the most significant margin between the classes is 
considered the best. Margin stands for the slice's greatest width parallel to the $H$ that has no inner data points. The data points, which are neighbouring to the separating $H$, are called support vectors (see Figure 2 ).

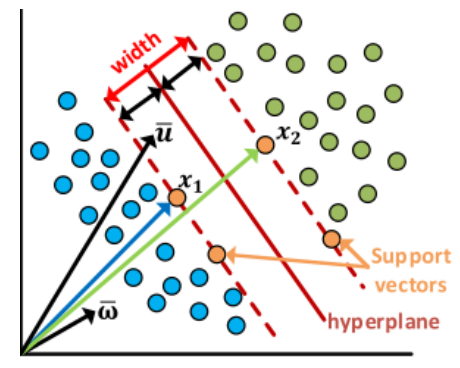

Figure 2. A sketch of the SVM [27]

Typically, the optimal $\mathrm{H}$ for p-dimensional features, from observations $(x)$, where $x=$ $\left\{x_{1}, x_{2}, \ldots, x_{n}\right\}$ with class labels $\left(y_{i}\right),\{i=1,2,3, \cdots, n \in[1,-1]\}$ is estimated as defined in (1) using a weight vector $\left(w^{t}\right)$ of the feature and a scalar of intercept $\left(\beta_{0}\right)$. Based on this $H$, the decision boundary between two class labels is computed using (2) and (3) as defined in [12], [19].

$$
\begin{aligned}
& f(x)=\left\{W^{t} x_{i}+\beta_{o}\right\} \\
& y_{i}\left(W^{t} x_{i}+\beta_{0} \geq+1 \text { if } y_{i}=1\right) \\
& y_{i}\left(W^{t} x_{i}+\beta_{0} \leq-1 \text { if } y_{i}=-1\right) \quad \forall_{i}=1,2, \ldots, n
\end{aligned}
$$

where $\mathrm{W}^{t}$ is a weight vector and $\beta_{0}$ is called the bias value

The SVM employs a practical mathematical function known as kernel trick to map the classification data and a dot-product for mapping a higher dimension. Usually, KFs are grouped into two classes, namely (i) rotation invariant kernel and (ii) translation invariant kernel. Table 1: shows the kernel functions for setting up an SVM model as defined [12].

Table 1. Types of KS for setting up an SVM model [12]

\begin{tabular}{lll}
\hline & \multicolumn{1}{c}{ Kernels } & $K\left(x_{i}, x_{j}\right)=1+x_{i}^{T} x_{j}$ \\
\hline 1. & Linear & $K\left(x_{i}, x_{j}\right)=\exp \left(1+x_{i}^{T} x_{j}\right)^{p}$ \\
2. & Polynomial & $K\left(x_{i}, x_{j}\right)=\exp \left(-\gamma\left\|x_{i}-x_{j}\right\|^{2}\right)$ \\
3. & Radial Basis Function (RBF) & $K\left(x_{i}, x_{j}\right)=\exp \left(\frac{-\left\|x_{i}-x_{j}\right\|^{2}}{2 \sigma^{2}}\right)$ \\
4. & Gaussian RBF & $K\left(x_{i}, x_{j}\right)=\exp \left(\frac{-\left\|x_{i}-x_{j}\right\|}{2 \sigma^{2}}\right)$ \\
& & $K\left(x_{i}, x_{j}\right)=\tanh \left(k x_{i}^{T} x_{j}-\delta\right)$ \\
5. & Exponential RBF & $k_{\text {spline }}(u, v)=1+u v+\int_{0}^{1}(u-t)_{+}(v-t)_{+} d t$ \\
6. & Sigmoid & $K(u, v)=1+k\left(u^{1}, v^{1}\right)+k\left(u^{2}, v^{2}\right)+k\left(u^{1}, v^{1}\right) k\left(u^{2}, v^{2}\right)$ \\
7. & Spline & $K(u, v)=\sum_{i}^{n} K(u, v)$ \\
8. & Anova Spline & \\
9. & Additive & $K(u, v)=\prod_{m-1}^{n} K_{m}\left(u_{m}, v_{m}\right)$ \\
10. & Tensor product & \\
& &
\end{tabular}

Lately, the SVM has gain popularity among SL algorithms for solving real-world problems, like handwriting analysis, facial analysis and more, specifically for pattern classification, outlier detection and regression-based applications [28], [29]. However, one critical challenge faced by SVM users is choosing the appropriate $\mathrm{KF}$ and its associated parameters, such as penalty parameter $(\mathrm{C}), \mathrm{KF}$ parameters like the gamma $(\gamma)$ for the RBF kernel. Studies [4], [17], [28] affirms that is the vital step in managing a learning task with the SVM since it has a substantial effect on its accuracy. Thus, the primary problems accompanying setting up the SVM model are how to select the KF and its allied parameter values for a specific domain application [4], [17], [28], and this paper attempts to find the best expression for these SVM parameters in three different 
domain. Specifically, we examine how the SVM efficiency in predicting diabetes, fake banknote, flower species and wheat species is affected by different KF and other parameters. Thus, we aim to make the KFs generalised for a specific domain. It is anticipated that this study outcome will serve as a ground base for several researchers (precisely beginners) to adopt the appropriate SVM parameter for a particular domain application without worrying.

\subsection{Study framework}

Figure 3 shows the experimental framework of this study. In (5) different data sets were downloaded from various sources for this study; see Table 2 for details.

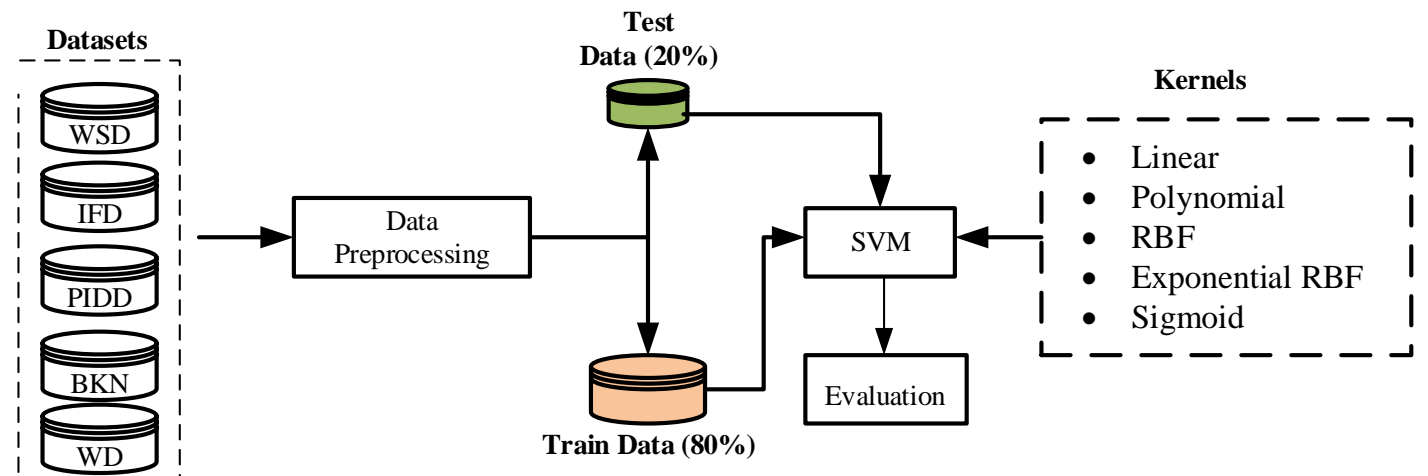

Figure 3. Study framework

Table 2. Used datasets details

\begin{tabular}{|c|c|c|c|c|}
\hline Dataset & $\begin{aligned} \text { Size } \\
(n \times n\end{aligned}$ & & Description & Source \\
\hline $\begin{array}{l}\text { Wheat seeds dataset } \\
\text { (WSD) }\end{array}$ & 210 & 8 & $\begin{array}{l}\text { The WDS contains measures of different wheat species. The aim is to predict a } \\
\text { seed species of a given seed feature. }\end{array}$ & A \\
\hline Iris flowers dataset (IFD) & 150 & 5 & $\begin{array}{l}\text { The IFD contains the measured features of the iris, and the aim is to predict a } \\
\text { flower species based on measured characteristics. }\end{array}$ & A \\
\hline $\begin{array}{l}\text { Pima indians diabetes } \\
\text { dataset (PIDD) }\end{array}$ & 768 & 9 & The PIDD is meant for predicting the inception of diabetes within 5-years. & B \\
\hline Banknotes (BKN) & 1372 & 5 & $\begin{array}{l}\text { The BKN aim at predicting the authenticity of a given banknote based on } \\
\text { extracted features }\end{array}$ & A \\
\hline Weather data (WD) & 14668 & 5 & $\begin{array}{l}\text { The weather data aims at predicting the maximum temperature using some } \\
\text { weather parameters }\end{array}$ & $\mathrm{C}$ \\
\hline
\end{tabular}

Firstly, we preprocessed each dataset separately to free the datasets from missing values, outliers and data inconsistency. We then normalised each dataset using the max-min function (see (4)) within the range $[0,1]$.

$$
x^{\prime}=\frac{x-x_{\min }}{x \min _{\max }}
$$

where $x^{\prime}$ is the normalisation value; $x=$ the value to be normalised, $x_{\min }$ and $x_{\max }$ are the minima and maxima value of the dataset.

Each normalised dataset is divided into a training set $(80 \%)$ and a testing set $(20 \%)$. From the different Kernels discussed in Table 1, we adopted five (5) most commonly used, namely: (i) linear, (ii) polynomial, (iii) radial basis function (RBF), (iv) exponential RBF and (v) sigmoid. Based on these six kernels. We set up an SVM model using different parameters (see Table 3). The aim is to make the kernels generalised for every dataset. 10-fold cross-validation was used for generalisation. We evaluate the performance of the SVM based on (i) confusion matrix, (ii) accuracy for classification analysis and root-mean-square-error (RMSE) for regression analysis. The Scikit-learn library was used to implement the SVM model. The experiment was conducted on Google colab [30], a free online platform for modelling ML models on powerful hardware options like GPU and TPU. Table 3 shows the details of the parameter used in modelling the SVM. 
Table 3. Used parameters

\begin{tabular}{ll}
\hline \multicolumn{1}{c}{ Parameter } & \multicolumn{1}{c}{ Description } \\
\hline Kernel & Represent the kernel type for algorithm \\
Penalty parameter $(C)$ & The Regularisation parameter, its strength is inversely proportional to C. \\
Degree $(d)$ & Degree of the polynomial kernel function ('poly'). All other kernels ignore it. \\
Gamma $(\gamma)$ & Kernel coefficient for 'radial basis function', 'polynomial' and 'sigmoid'. \\
\hline
\end{tabular}

\section{EXPERIMENTAL RESULTS AND DISCUSSION}

Table 4 shows the experimental results of different KF with several datasets. The results show that one single KF is not suitable for all domains, which confirms arguments by literature [4], [17], [28] that selecting the correct KF is a vital step in setting up an SVM model. An incorrect choice of the KF will lead to abysmal results by the model. Hence, the random selection of KF is not always optimal for achieving a high SVM generalisation. From the outcome, it suggests that the linear kernel is more suitable for a small dataset. Also, SVM is not a parametric model, so complexity increases as the training dataset's size increases.

Table 4 shows that the optimal kernels effectively enhance the SVM prediction performance and generalizability by optimising the result. Likewise, the same kernel function on different datasets gave different results (see Table 4); this suggests that a given dataset's statistical property can effectively inform the choice of a KF and its parameters.

Figures 4-9 shows the confusion matrix plot for each KF and its parameters on the different datasets; the results affirm Table 4. From Table 4 and Figures 4-9, it can be inferred that the choice of an SVM KF highly depends on the problem at hand, i.e., what one is attempting to model. Therefore, the motivation behind selecting a specific KF is very intuitive and upfront based on what kind of information one is intended to extract about the dataset. The outcome (see Table 4) affirms that correct tuning of the KF parameter in the RBF, Sigmond and exponential RBF KFs increases SVM accuracy compared with the linear kernel.

Table 4. Comparing different kernels on datasets

\begin{tabular}{|c|c|c|c|c|c|c|}
\hline \multirow{3}{*}{\multicolumn{2}{|c|}{ Kernel Function (Associated Parameters) }} & \multicolumn{5}{|c|}{ Machine Learning Task } \\
\hline & & \multirow{2}{*}{\multicolumn{4}{|c|}{$\begin{array}{l}\text { Classification } \\
\text { Accuracy (\%) }\end{array}$}} & \multirow{3}{*}{$\begin{array}{c}\text { Regression } \\
\text { RMSE } \\
\text { WD }\end{array}$} \\
\hline & & & & & & \\
\hline & & IFD & \multirow{2}{*}{$\begin{array}{c}\text { PIDD } \\
76.6\end{array}$} & \multirow{2}{*}{$\begin{array}{c}\text { WSD } \\
\mathbf{9 5}\end{array}$} & \multirow{2}{*}{$\begin{array}{c}\text { BKN } \\
99\end{array}$} & \\
\hline \multirow[b]{2}{*}{ Linear } & $\mathrm{C}=5$ & 80 & & & & 0.22 \\
\hline & $\mathrm{C}=100000$ & 80 & 75 & 95 & 99 & 0.22 \\
\hline \multirow{2}{*}{ Polynomial } & $\mathrm{C}=5, \mathrm{~d}=2$ & 76.7 & 64.9 & 68 & 75 & 1.02 \\
\hline & $\mathrm{C}=100000, \mathrm{~d}=3$ & 66.7 & 68 & 57 & 87 & 1.01 \\
\hline \multirow{2}{*}{$\mathrm{RBF}$} & $\mathrm{C}=5, \gamma=5$ & 70 & 70.8 & 92 & 100 & 0.19 \\
\hline & $\mathrm{C}=100000, \gamma=2$ & 73.3 & 70 & 88 & 100 & 0.16 \\
\hline \multirow[t]{2}{*}{ Sigmoid } & $\mathrm{C}=5, \mathrm{k}=0.5, \delta=0$ & 43.3 & 70.2 & 90 & 75 & 1668.3 \\
\hline & $\mathrm{C}=100000, \mathrm{k}=2, \delta=4$ & 43.3 & 70 & 88 & 75 & 1700.1 \\
\hline \multirow{2}{*}{ Exponential RBF } & $\mathrm{C}=5, \sigma=2$ & 76.7 & 77.4 & 93 & 99.6 & 0.2 \\
\hline & $\mathrm{C}=100000, \sigma=5$ & 73.3 & 77.38 & 93 & 99.62 & 0.19 \\
\hline
\end{tabular}
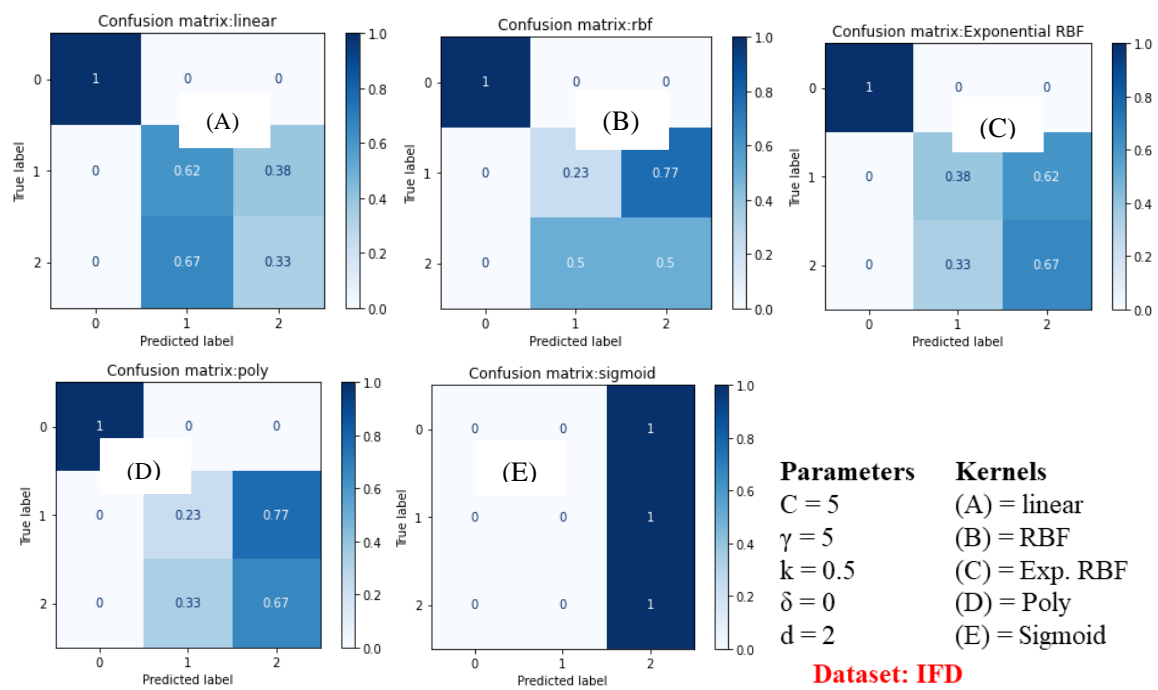

Figure 4. Confusion matrix of SVM with different kernels on IFD dataset 

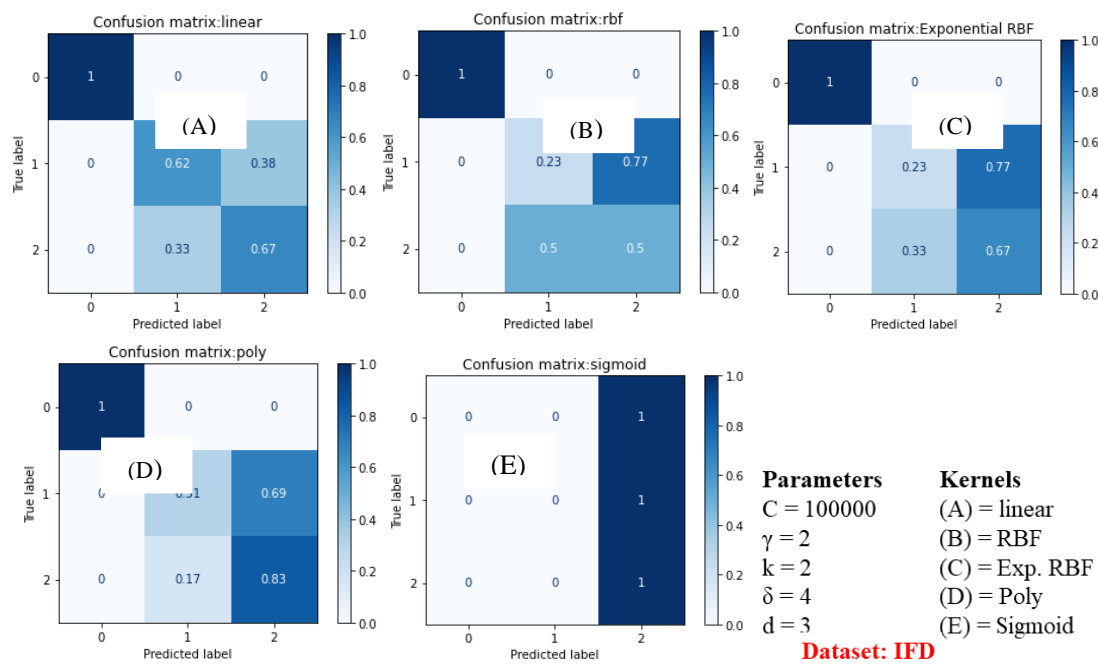

Figure 5. Confusion matrix of SVM with different kernels on PIDD dataset
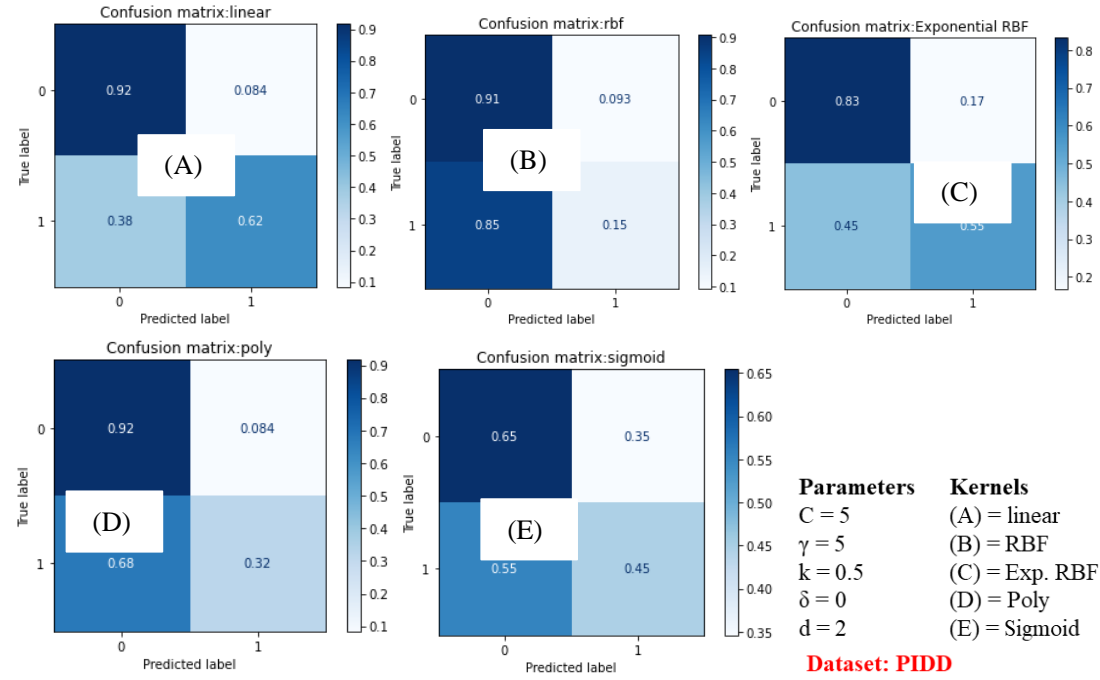

Figure 6. Confusion matrix of SVM with different kernels on PIDD dataset
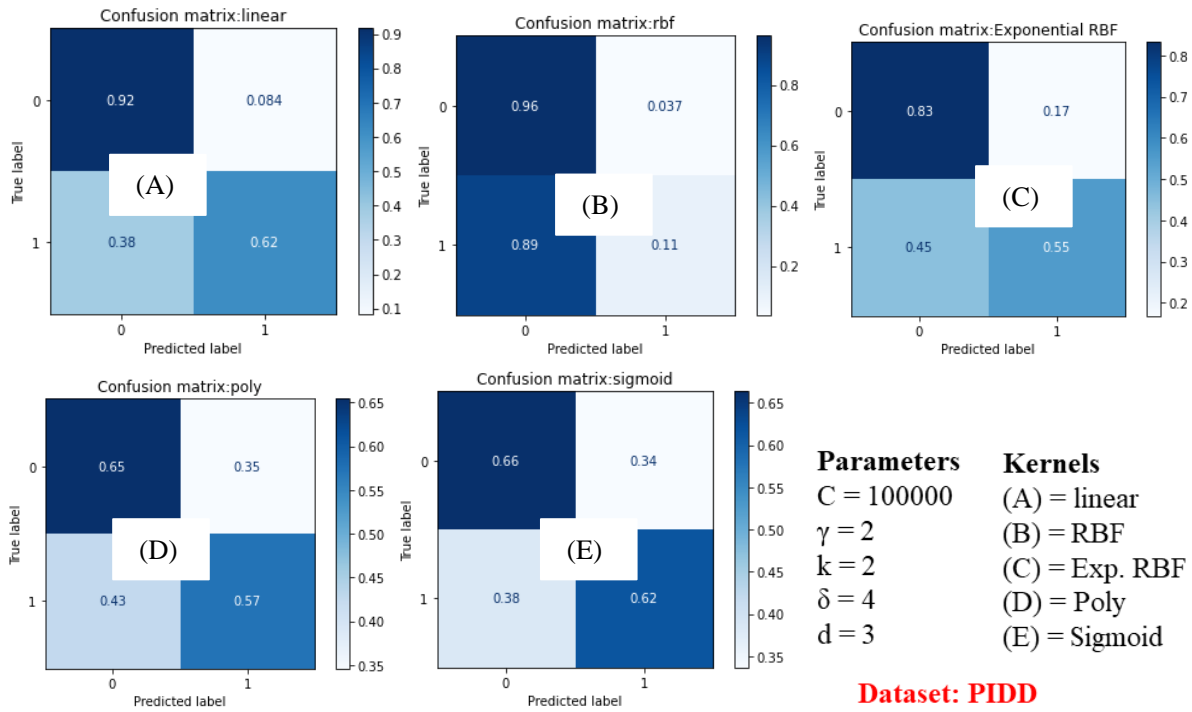
Parameters
Kernels
$\mathrm{C}=100000$
$(\mathrm{A})=$ linear
$\gamma=2$
$\mathrm{k}=2$
(B) $=\mathrm{RBF}$
(C) $=$ Exp. RBF
$\delta=4$
(D) $=$ Poly
(E) $=$ Sigmoid
Dataset: PIDD

Figure 7. Confusion Matrix of SVM with different kernels on PIDD dataset 

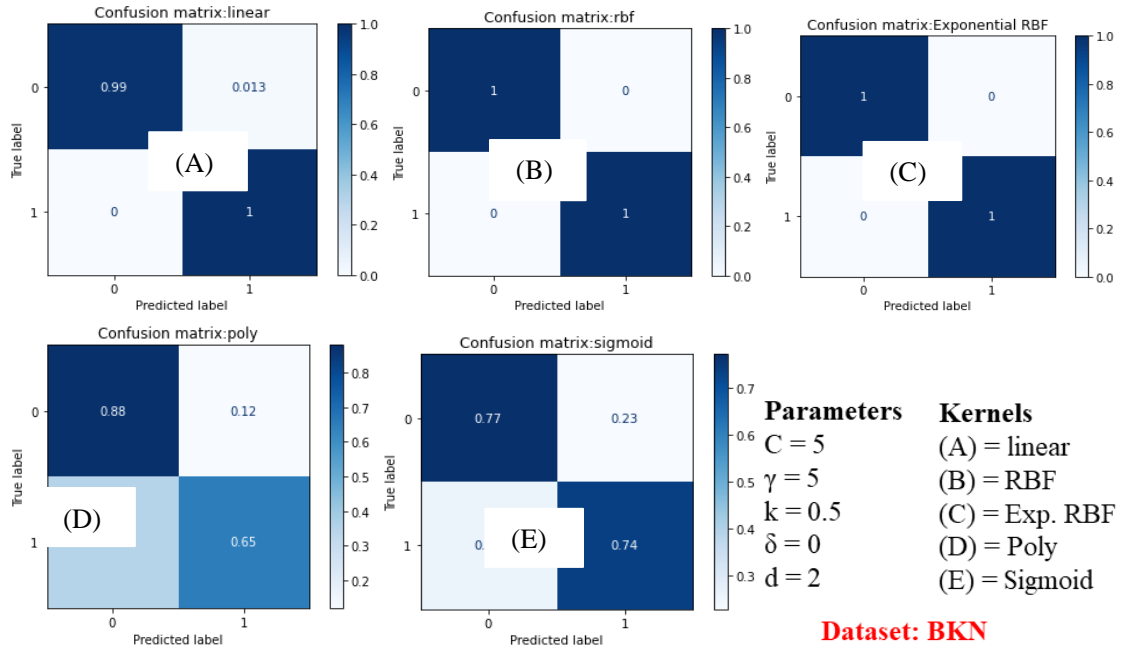

Parameters

Kernels

(A) $=$ linear

(B) $=\mathrm{RBF}$

(C) $=$ Exp. RBF

(D) $=$ Poly

(E) $=$ Sigmoid

Figure 8. Confusion matrix of SVM with different kernels on BKN dataset
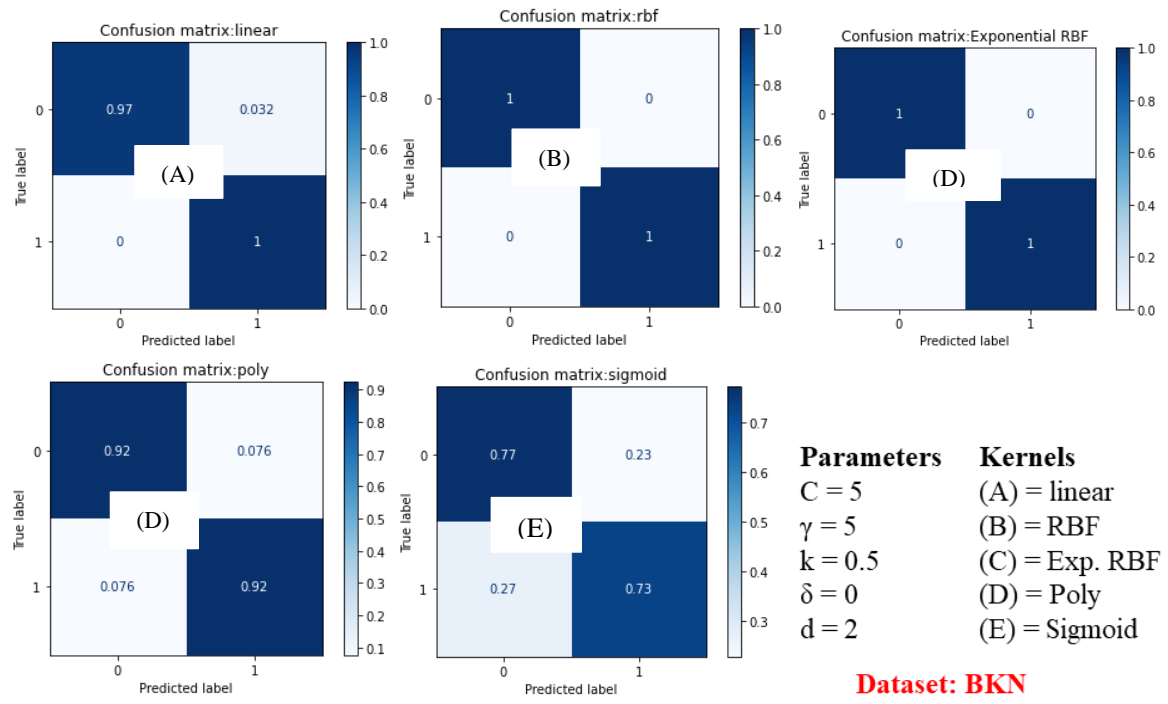
Parameters
$\mathrm{C}=5$
Kernels
$\gamma=5$
$\mathrm{k}=0.5$
$\delta=0$
$d=2$
(A) = linea
(B) $=\mathrm{RBF}$
(C) $=$ Exp. RBF
(D) $=$ Poly
(E) $=$ Sigmoid

Figure 9. Confusion matrix of SVM with different kernels on BKN dataset

\section{CONCLUSION}

SVM is one of the machine learning algorithms that has received much attention from the research committee and professionals. Its success has been seen in every sector of our day-to-day activities. On the other hand, one primary concern with its implementation is selecting the most appropriate kernel function and finetuning its associated parameters. Several KF can be used for different applications, but the most suitable depends on the problem at hand (domain area). This paper reviewed and examined the effect of the linear, polynomial, RBF, sigmoid, and exponential RBF Kernel functions on the SVM algorithm's performance. We assessed these KF performances on the SVM using the confusion matrix and accuracy for classification and the RMSE for regression tasks. It is observed that no single $\mathrm{KF}$ is suitable for all classification problems or regression problems. Also, an SVM with optimised kernel parameters for RBF and exponential RBF KFs are more likely to outperform the linear and sigmoid kernel base SVM methods in terms of accuracy. Since the KFs performance varies with the problem at hand, this study suggests a fused kernel method as a way forward. Hence, a systematic method and optimisation technique such as genetic algorithm (GA) and particle swarm optimisations (PSO) can be used to build a unified kernel. Therefore, the challenge of selecting the correct kernels and the best logical method of merging these kernels is a future direction.

\section{ACKNOWLEDGEMENTS}

We express our gratitude to everyone who contributed to the development of this paper. 


\section{REFERENCES}

[1] S. Natale and A. Ballatore, "Imagining the thinking machine: Technological myths and the rise of artificial intelligence," Convergence, vol. 26, no. 1, pp. 3-18, 2020, doi: 10.1177/1354856517715164.

[2] E. Farrow, "To augment human capacity-Artificial intelligence evolution through causal layered analysis," Futures, vol. 108, pp. 61-71, 2019, doi: 10.1016/j.futures.2019.02.022.

[3] I. K. Nti, A. F. Adekoya and B. A. Weyori, "A systematic review of fundamental and technical analysis of stock market predictions," Artificial Intelligence Review, vol. 53, no. 4, pp. 3007-3057, 2019, doi: 10.1007/s10462-01909754-z.

[4] I. K. Nti, A. F. Adekoya and B. A. Weyori, "Efficient Stock-Market Prediction Using Ensemble Support Vector Machine," Open Computer Science, vol. 10, no. 1, pp. 153-163, Jul. 2020, doi: 10.1515/comp-2020-0199.

[5] H. Jiang, W.-K. Ching, K. F. C. Yiu and Y. Qiu, "Stationary Mahalanobis kernel SVM for credit risk evaluation," Applied Soft Computing, vol. 71, pp. 407-417, Oct. 2018, doi: 10.1016/j.asoc.2018.07.005.

[6] M. Elangovan, V. Sugumaran, K. I. Ramachandran and S. Ravikumar, "Effect of SVM kernel functions on classification of vibration signals of a single point cutting tool," Expert Systems with Applications, vol. 38, no. 12, pp. 15202-15207, 2011, doi: 10.1016/j.eswa.2011.05.081.

[7] C. Venkatesan, P. Karthigaikumar, A. Paul, S. Satheeskumaran and R. Kumar, "ECG Signal Preprocessing and SVM Classifier-Based Abnormality Detection in Remote Healthcare Applications," in IEEE Access, vol. 6, pp. 9767-9773, 2018, doi: 10.1109/ACCESS.2018.2794346.

[8] K. Harimoorthy and M. Thangavelu, "Multi-disease prediction model using improved SVM-radial bias technique in healthcare monitoring system," Journal of Ambient Intelligence and Humanized Computing, vol. 12, pp. 37153723, 2020, doi: 10.1007/s12652-019-01652-0.

[9] M. Kathuria and S. Gambhir, "Critical Condition Detection Using Lion Hunting Optimizer and SVM Classifier in a Healthcare WBAN," International Journal of E-Health and Medical Communications, vol. 11, no. 1, pp. 52-68, 2020, doi: 10.4018/IJEHMC.2020010104.

[10] V. N. Vapnik, "An overview of statistical learning theory," in IEEE Transactions on Neural Networks, vol. 10, no. 5, pp. 988-999, Sept. 1999, doi: 10.1109/72.788640.

[11] M. E. Matheny, F. S. Resnic, N. Arora and L. Ohno-Machado, "Effects of SVM parameter optimisation on discrimination and calibration for post-procedural PCI mortality," Journal of Biomedical Informatics, vol. 40, no. 6, pp. 688-697, 2007, doi: 10.1016/j.jbi.2007.05.008.

[12] R. Sangeetha and B. Kalpana, "A Comparative Study and Choice of an Appropriate Kernel for Support Vector Machines," in Communications in Computer and Information Science, vol. 101, pp. 549-553, 2010, doi: 10.1007/978-3-642-15766-0_93.

[13] V.-H. Nhu et al., "Comparison of Support Vector Machine, Bayesian Logistic Regression, and Alternating Decision Tree Algorithms for Shallow Landslide Susceptibility Mapping along a Mountainous Road in the West of Iran,” Applied Sciences, vol. 10, no. 15, p. 5047, 2020, doi: 10.3390/app10155047.

[14] A. E. Minarno, W. A. Kusuma and H. Wibowo, "Performance Comparisson Activity Recognition using Logistic Regression and Support Vector Machine," in 2020 3rd International Conference on Intelligent Autonomous Systems (ICoIAS), Feb. 2020, pp. 19-24, doi: 10.1109/ICoIAS49312.2020.9081858.

[15] A. Sabat-Tomala, E. Raczko and B. Zagajewski, "Comparison of Support Vector Machine and Random Forest Algorithms for Invasive and Expansive Species Classification Using Airborne Hyperspectral Data," Remote Sensing, vol. 12, no. 3, p. 516, Feb. 2020, doi: 10.3390/rs12030516.

[16] C. Aroef, Y. Rivan and Z. Rustam, "Comparing random forest and support vector machines for breast cancer classification," TELKOMNIKA Telecommunication Computer Electron and Control, vol. 18, no. 2, p. 815, 2020, doi: 10.12928/telkomnika.v18i2.14785.

[17] I. K. Nti, A. F. Adekoya and B. A. Weyori, "A comprehensive evaluation of ensemble learning for stock-market prediction," Journal of Big Data vol. 7, no. 1, p. 20, 2020, doi: 10.1186/s40537-020-00299-5.

[18] J. Horak, J. Vrbka and P. Suler, "Support Vector Machine Methods and Artificial Neural Networks Used for the Development of Bankruptcy Prediction Models and their Comparison," Journal of Risk and Financial Management, vol. 13, no. 3, p. 60, 2020, doi: 10.3390/jrfm13030060.

[19] M. Yaqoob, F. Iqbal and S. Zahir, "Comparing predictive performance of k-nearest neighbors and support vector machine for predicting ischemic heart disease," Research Journal in Advanced Sciences, vol. 1, no. 2, pp. 49-60, 2020.

[20] S. Shabani et al., "Modeling Pan Evaporation Using Gaussian Process Regression K-Nearest Neighbors Random Forest and Support Vector Machines; Comparative Analysis," Atmosphere, vol. 11, no. 1, p. 66, 2020, doi: 10.3390/atmos11010066.

[21] I. S. Al-Mejibli, D. H. Abd, J. K. Alwan and A. J. Rabash, "Performance Evaluation of Kernels in Support Vector Machine," 20181 st Annual International Conference on Information and Sciences (AiCIS), 2018, pp. 96-101, doi: 10.1109/AiCIS.2018.00029.

[22] B. Peng, L. Peng, G. Dao-tian and L. Yan, "Study on SVM Calibration Model Parameter for Mixed Gas," Procedia Engineering, vol. 15, pp. 3642-3645, 2011, doi: 10.1016/j.proeng.2011.08.682.

[23] T. Mitchell, "Machine Learning," 1st Editio. McGraw Hill, 1997.

[24] R. V. Molina, "Choosing the Right Kernel a Meta-Learning Approach To Kernel Selection in Support Vector Machines Choosing the Right Kernel a Meta-Learning Approach To Kernel," University of Houston, 2015.

[25] I. K. Nti, A. F. Adekoya, B. A. Weyori and O. Nyarko-Boateng, "Applications of artificial intelligence in engineering and manufacturing: a systematic review," Journal of Intelligent Manufacturing, pp. 1-29, 2021, doi: 
10.1007/s10845-021-01771-6.

[26] I. K. Nti, M. Teimeh, O. Nyarko-Boateng and A. F. Adekoya, "Electricity load forecasting: a systematic review," Journal of Electrical Systems and Information Technology, vol. 7, no. 13, pp. 1-19, Dec. 2020, doi: 10.1186/s43067-020-00021-8.

[27] C. Savas and F. Dovis, "Comparative Performance Study of Linear and Gaussian Kernel SVM Implementations for Phase Scintillation Detection," 2019 International Conference on Localization and GNSS (ICL-GNSS), 2019, pp. 16, doi: 10.1109/ICL-GNSS.2019.8752635.

[28] S. Sarafrazi and H. Nezamabadi-pour, "Facing the classification of binary problems with a GSA-SVM hybrid system," Mathematical and Computer Modelling, vol. 57, no. 1-2, pp. 270-278, 2013, doi: 10.1016/j.mcm.2011.06.048.

[29] L. C. Padierna, M. Carpio, A. Rojas-Domínguez, H. Puga and H. Fraire, "A novel formulation of orthogonal polynomial kernel functions for SVM classifiers: The Gegenbauer family," Pattern Recognition, vol. 84, pp. 211225, Dec. 2018, doi: 10.1016/j.patcog.2018.07.010.

[30] E. Bisong, "Building Machine Learning and Deep Learning Models on Google Cloud Platform," Google Colaboratory, Berkeley, CA: Apress, 2019, pp. 59-64.

\section{BIOGRAPHIES OF AUTHORS}
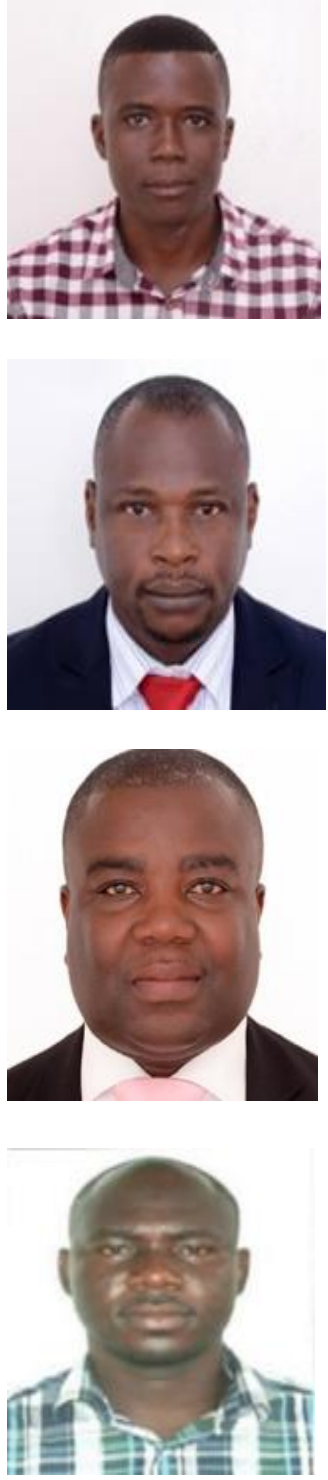

Isaac Kofi Nti holds HND in Electrical \& Electronic Engineering from Sunyani Technical University, B. Sc. in Computer Science from Catholic University College, M. Sc. in Information Technology from Kwame Nkrumah University of Science and Technology. Mr $\mathrm{Nti}$ is a Lecturer at the Department of Computer Science and Informatics, University of Energy and Natural Resources (UENR), Sunyani Ghana and currently is a Ph. D. candidate and the same department. His research interests include artificial intelligence, energy system modelling, intelligent information systems, social and sustainable computing, business analytics, and data privacy and security. Email: Isaac.nti@uenr.edu.gh. ORCID ID: https://orcid.org/0000-0001-9257-4295

Adebayo Felix Adekoya holds B. Sc. (1994), M. Sc. (2002), and Ph. D. (2010) in Computer Science, an MBA in Accounting \& Finance (1998), and a Postgraduate Diploma in Teacher Education (2004). In addition, he has put in about twenty-five (25) years of experience as a lecturer, researcher and administrator at the higher educational institution levels in Nigeria and Ghana. A. F. Adekoya is an Associate Professor of Computer Science. Currently, he serves as the Dean, School of Sciences, and the Acting Pro-VC University of Energy and Natural Resources, Sunyani, Ghana. His research interests include artificial intelligence, business \& knowledge engineering, intelligent information systems, and social and sustainable computing. ORCID iD: https://orcid.org/0000-0002-5029-2393

Owusu Nyarko-Boateng is a PhD Computer Science candidate and a lecturer at the Department of Computer Science and Informatics, the University of Energy and Natural Resources, Ghana. He holds HND Electrical \& Electronics Engineering, BSc Computer Science, PGDE, and MSc Information Technology. He has in-depth experience in telecommunications transmission systems, including fiber optics cable deployment for a longhaul and short distance (FTTx), 2G BTS, WCDMA (3G), and 4G (LTE) plants installations and configurations. His research areas include machine learning, artificial intelligence, computer networks and data communications, network security, fiber optics technologies, modelling transmission systems, 5G \& 6G Technologies, Expert Systems, computational intelligence for data communications.

Benjamin Asubam Weyori received his Ph. D. and M. Phil. in Computer Engineering from the Kwame Nkrumah University of Science and Technology (KNUST), Ghana, in 2016 and 2011, respectively. He obtained his Bachelor of Science in Computer Science from the University for Development Studies (UDS), Tamale, Ghana, in 2006. He is currently a Senior Lecturer and the Head of the Department of Computer Science and Informatics, the University of Energy and Natural Resources (UENR) in Ghana. His main research interests include artificial intelligence, computer vision (image processing), machine learning, and web engineering. ORCID iD: https://orcid.org/0000-0001-5422-425 